\title{
Infinite hierarchies of nonlinearly dependent periodic orbits
}

\author{
Jason A. C. Gallas* \\ Departamento de Física, Faculdade de Ciências, Universidade de Lisboa, 1749-016 Lisboa, Portugal \\ and Instituto de Física, Universidade Federal do Rio Grande do Sul, 91501-970 Porto Alegre, Brazil \\ (Received 2 May 2000; revised manuscript received 14 September 2000; published 27 December 2000)
}

\begin{abstract}
Quadratic maps are used to show explicitly that the skeleton of unstable periodic orbits underlying classical and quantum dynamics is stratified into a doubly infinite hierarchy of orbits inherited from a set of basic "seeds" through certain nonlinear transformations $T_{\alpha}(x)$. The hierarchy contains nonunique substructurings which arise from the different possibilities of sequencing the transformations $T_{\alpha}(x)$. The structuring of the orbital skeleton is shown to be generic for Abelian equations, i.e., for all dynamical systems generated by iterating rational functions.
\end{abstract}

DOI: 10.1103/PhysRevE.63.016216

PACS number(s): 05.45.- a, 03.65.Fd, 45.05.+x

The skeleton of unstable periodic orbits (UPOs) in chaotic attractors is by now a well-recognized and studied subject, yet it still poses great challenges in mathematical physics: to establish reliable algorithms allowing efficient location of the skeleton [1] or criteria allowing one to know when the infinite skeleton of unstable orbits may be replaced by only the lowest period orbits while still providing a useful approximation of the chaotic attractor [2], to name only two. In fact, one may argue [3] that the finding that UPOs provide a skeleton for the organization of the very complex chaotic dynamics can be considered one of the major advances in the understanding of the behavior of nonlinear dynamical systems during the past ten years. In a different setting, the Gutzwiller trace formula links the eigenvalue of the Schrödinger operator as Planck's constant goes to zero (the semiclassical limit) with the closed orbits of the corresponding classical mechanical system [4-6]. So, periodic orbits possess numerous applications in different areas of physics.

Extending the seminal works of Sharkovskii [7] and Li and Yorke [8] on the coexistence of cycles of a continuous map of the line into itself [9] and of Thurston [10] on the geometry and dynamics of diffeomorphisms, modern works on the organization and hierarchies of UPOs have been worked out by Gambaudo et al. [11] and by Hall [12] on one-dimensional representatives of pseudo Anosov isotopy classes with minimal periodic orbit structure.

Although applications of periodic orbits invariably assume them to be independent from each other, we found recently [13] an interesting phenomenon of "inheritance" which interconnects periodic orbits. Inheritance means orbits within orbits: applying nonlinear transformations to certain $k$-periodic "mother" orbits yields several additional $k$-periodic "daughter" orbits. For instance, for the quadratic map the transformations

$$
T_{3}(x)=x^{3}-3 x \quad \text { and } \quad T_{5}(x)=x^{5}-5 x^{3}+5 x
$$

triplicate and quintuplicate the quantity of periodic orbits. However, inheritance was previously found to occur only among a few very specific orbits, all isoperiodic [14].

*Email address: http://www.if.ufrgs.br/ jgallas
In the present paper we show explicitly the existence of infinite sets of nonlinear transformations $T_{\alpha}(x)$ interconnecting orbits of distinct periodicities. In addition, we are also able to elucidate the origin of this phenomenon, showing orbital interdependencies to be a generic property of "Abelian equations" [15], i.e., of equations of motion having their roots expressible as rational functions of one of them. This result is important because every dynamical system obtained by iterating arbitrary [16] rational functions $f(x)$ has its periodic orbits defined necessarily by Abelian equations since their roots are all interconnected $a b$ ovo by the recurrence $x_{t+1}=f\left(x_{t}\right)$ defining the system. Thus, orbital inheritance is a generic property of orbital equations generated by iterating rational functions, a rather large class.

The same transformations $T_{3}(x)$ and $T_{5}(x)$ found previously to interconnect isoperiodic orbits are now shown to also interconnect orbits of different periodicities. In fact, $T_{3}(x)$ and $T_{5}(x)$ are among the first members of an infinite set $\left\{T_{\alpha}(x)\right\}$ of transformations which play a much more fundamental and general role than previously anticipated. So, equipped with $\left\{T_{\alpha}(x)\right\}$, it is possible to uncover a remarkable doubly infinite hierarchical stratification of the skeleton of unstable periodic orbits of the quadratic map, closely resembling a spectral decomposition. This stratification is a natural consequence of the repeated application of $T_{\alpha}(x)$ to the orbital points and it is expected to be of relevance for the semiclassical interpretations of atomic spectra with trace formulas involving the skeleton of UPOs [4-6].

We start by generalizing the results of Ref. [13]. Iterating the quadratic map $f(x)=2-x^{2}$ one obtains a family of polynomial factors $P_{k}(x)$ containing all genuine motions of period $k$ and only these. The procedure for decomposing physical trajectories into such "prime" factors $P_{k}(x)$ is described in Ref. [13]. So, the quadratic map has two period-1 orbits, defined by the roots of $P_{1}(x)$, one period-2 orbit defined by $P_{2}(x)$, two period-3 orbits defined by $P_{3}(x)$ $=P_{3,1}(x) P_{3,2}(x)$, three period-4 orbits defined by $P_{4}(x)$ $=P_{4,1}(x) P_{4,2}(x)$, etc. where

$$
\begin{gathered}
P_{1}(x)=x^{2}+x-2=(x+2)(x-1), \quad \Delta=3^{2}, \\
P_{2}(x)=x^{2}-x-1, \quad \Delta=5,
\end{gathered}
$$




$$
\begin{gathered}
P_{3,1}(x)=x^{3}-3 x-1, \quad \Delta=3^{4}, \\
P_{3,2}(x)=x^{3}-x^{2}-2 x+1, \quad \Delta=7^{2} \\
P_{4,1}(x)=x^{4}+x^{3}-4 x^{2}-4 x+1, \quad \Delta=3^{5} 5^{3}, \\
P_{4,2}(x)=x^{8}-x^{7}-7 x^{6}+6 x^{5}+15 x^{4}-10 x^{3}-10 x^{2}+4 x+1, \\
\Delta=17^{7} .
\end{gathered}
$$

The subindex $k$ of $P_{k, j}(x)$ indicates the period while $j$ labels different factors, if necessary [17]. $\Delta$ is the discriminant [18] of each polynomial. The sequence of $P_{k, j}(x)$ is infinite in $k$ and the zeros of every $P_{k, j}(x)$ define one or more $k$-periodic orbit. We call “orbital equation" any polynomial defining just a single orbit, with coefficients rational or not, and a "class equation" those with coefficients necessarily rational and with zeros defining more than one isoperiodic orbit. Thus, $P_{4,2}(x)$ is a class equation composed by two conjugate orbital equations:

$$
\begin{aligned}
P_{4,2}(x)= & {\left[x^{4}-(1+\sqrt{17}) x^{3} / 2-(3-\sqrt{17}) x^{2} / 2\right.} \\
& -(2-\sqrt{17}) x-1] \times\left[x^{4}-(1-\sqrt{17}) x^{3} / 2\right. \\
& \left.-(3+\sqrt{17}) x^{2} / 2-(2+\sqrt{17}) x-1\right] .
\end{aligned}
$$

Here we deal only with orbital equations.

Now, consider the family $W_{n}(x)$ of polynomials, generated recursively as follows:

$$
W_{n}(x)=W_{n-1}\left[T_{\alpha}(x)\right], \quad W_{0}(x)=P_{k, j}(x),
$$

$n=1,2,3 \ldots$ Every sequence $W_{n}(x)$ generated by iterating Eq. (8) depends on (i) an initial seed $P_{k, j}(x)$, and (ii) a transformation $T_{\alpha}(x)$. Straightforward calculations show that every polynomial $W_{n}(x)$ may be splitted into a product of (i) a trivial factor, i.e., a factor which is already known, essentially $W_{n-1}(x)$ and $P_{k, j}(x)$ itself, and (ii) a nontrivial factor, denoted ad hoc by $D_{n}(x)$, the daughters. Thus, $W_{n}(x)=D_{n}(x) \times[$ trivial factors $]$. All zeros of $D_{n}(x)$ are orbital points of the inherited trajectories.

The daughters $D_{n}(x)$ may be easily extracted from $W_{n}(x)$ by polynomial division, implemented as a recurrence relation:

$$
D_{n}(x)=\frac{W_{n}(x)}{D_{n-1}(x) W_{n-1}(x)}, \quad n=1,2,3, \ldots,
$$

where $D_{0}(x)=1$ with one exception [19]. Since modulo trivial factors $D_{n}(x)$ coincide with $W_{n}(x), D_{n}(x)$ is built from the same "parent" quantities as $W_{n}(x)$, namely, $P_{k, j}(x)$ and $T_{\alpha}(x)$. When parenthood is an issue, we use $D_{n}^{k^{J}, \alpha}(x)$ to mark inheritance from $P_{k, j}(x)$ and $T_{\alpha}(x)$. Since $D_{n}(x)$ and $W_{n}(x)$ are Abelian equations, their zeros may be obtained by finite sequences of radicals. Now, we exhibit some elements of $\left\{D_{n}^{k^{j}, \alpha}(x)\right\}$ explicitly.

Table I shows the first few members for two infinite hierarchies $D_{n}(x)$, obtained by starting from $P_{1}(x)$ and from $P_{2}(x)$ and tripling the period with $T_{3}(x)$. These polynomials
TABLE I. The first members of the infinite hierarchies of $k$-periodic orbits for $k=3^{n}$ and $k=4 \times 3^{n-1}$ inherited from $P_{1}(x)$ $=x^{2}+x-2$ and from $P_{2}(x)=x^{2}-x-1$ by tripling the period with $T_{3}(x)$. One orbital point for every $3^{n}$-periodic orbit is given by $2 \cos \left(\pi / 3^{n+1}\right)$. The discriminant $\Delta$ of each hierarchy has the same structure.

\begin{tabular}{lrll}
\hline \hline$n$ & \multicolumn{1}{c}{$k$} & $D_{n}^{1,3}(x)$, inherited from $P_{1}\left[T_{3}(x)\right]$ & \multicolumn{1}{c}{$\Delta$} \\
\hline 1 & 3 & $x^{3}-3 x-1$ & $3^{4}$ \\
2 & 9 & $x^{9}-9 x^{7}+27 x^{5}-30 x^{3}+9 x-1$ & $3^{22}$ \\
3 & 27 & $x^{27}-27 x^{25}+\cdots-27 x-1$ & $3^{94}$ \\
4 & 81 & $x^{81}-81 x^{79}+\cdots+81 x-1$ & $3^{364}$ \\
5 & 243 & $x^{243}-243 x^{241}+\cdots-243 x-1$ & $3^{1336}$ \\
$l$ & $3^{l}$ & $x^{3^{l}}-3^{l} x^{3^{l}-2}+\cdots+(-3)^{l} x-1$ & \\
& & & \\
$n$ & $k$ & $D_{n}^{2,3}(x)$, inherited from $P_{2}\left[T_{3}(x)\right]$ & $\Delta$ \\
\hline 1 & 4 & $x^{4}+x^{3}-4 x^{2}-4 x+1$ & $3^{2} 5^{3}$ \\
2 & 12 & $x^{12}-12 x^{10}+x^{9}+\cdots+12 x+1$ & $3^{18} 5^{9}$ \\
3 & 36 & $x^{36}-36 x^{34}+\cdots-36 x+1$ & $3^{90} 5^{27}$ \\
4 & 108 & $x^{108}-108 x^{106}+\cdots+108 x+1$ & $3^{378} 5^{81}$ \\
5 & 324 & $x^{324}-324 x^{322}+\cdots-324 x+1$ & $3^{1458} 5^{243}$ \\
\hline \hline
\end{tabular}

are irreducible over the rationals. Since all orbits here are defined by real numbers, this irreducibility implies that such polynomials are already the simplest normal forms, the minimum polynomials [18] which fix, slave, the arithmetical properties of orbital equations and its solutions. A useful quantity in this context is the multiplicity $\mu$ of every polynomial of the family $\left\{D_{n}(x)\right\}$, defined by $\mu=\delta / k$, where $\delta$ is the degree of the polynomial and $k$ is the period of its orbits. The multiplicity is a characteristic integer informing the quantity of $k$-periodic orbits defined by the zeros of the irreducible factors $D_{n}(x)$, i.e., the order of the class.

As shown in Table I, $P_{1}\left[T_{3}(x)\right]$ generates orbits with period $3^{n}$ while $P_{2}\left[T_{3}(x)\right]$ generates orbits with period 4 $\times 3^{n-1}$, the multiplicity of all orbits being $\mu=1$, meaning that each polynomial is an orbital equation.

A remarkable property shared by all daughters $D_{n}(x)$, here and later on, is that they are themselves factors defining periodic orbits, i.e., all their zeros are periodic points of the system. Therefore, every daughter gives rise to a new hierarchy of orbits, implying that each daughter may be used as a new transformation $T_{\alpha}(x)$ to produce additional orbits, $a d$ infinitum.

The hierarchy inherited from $P_{3,1}\left[T_{3}(x)\right]$ is a subset, a substructure, of that inherited from $P_{1}\left[T_{3}(x)\right]$, i.e., $D_{n}^{3^{1}, 3}(x)=D_{n+1}^{1,3}(x) . \quad$ Analogously, $\quad D_{n}^{3^{1}, 9}(x)=D_{2 n-1}^{1,3}(x)$ where $T_{9}(x)=T_{3}\left[T_{3}(x)\right]$. An infinity of similar hierarchical substructurings exist. They are simply harmonics of their original orbit.

Table II shows the first few members of three additional hierarchies, namely, $D_{n}^{3,2}(x), D_{n}^{4,5}(x)$, and $D_{n}^{5,1}(x)$. Now, essentially all orbits have multiplicity $\mu \neq 1$, meaning that each irreducible polynomial defines $\mu$ orbits of period $k$. As before, discriminants display remarkably simple structures, being fast-growing powers of small prime numbers. The actual 
TABLE II. The first few members of three hierarchies inherited by tripling the period of $P_{3,2}(x), P_{4,2}(x)$, and $P_{5,1}(x)$. These hierarchies start from reciprocal-looking polynomials, i.e., polynomials having pairs of identical coefficients. Most polynomials are class equations. Notice the regularities of $\Delta$ within a family.

\begin{tabular}{|c|c|c|c|c|}
\hline$n$ & $k$ & $\mu$ & $D_{n}^{3^{2}, 3}(x)$, inherited from $P_{3,2}\left[T_{3}(x)\right]$ & $\Delta$ \\
\hline 1 & 6 & 1 & $x^{6}+x^{5}-6\left(x^{4}+x^{3}\right)+8\left(x^{2}+x\right)+1$ & $3^{3} 7^{5}$ \\
\hline 2 & 6 & 3 & $x^{18}-18 x^{16}+\cdots-24 x+1$ & $3^{27} 7^{15}$ \\
\hline 3 & 18 & 3 & $x^{54}-54 x^{52}+\cdots+72 x+1$ & $3^{135} 7^{45}$ \\
\hline 4 & 54 & 3 & $x^{162}-162 x^{160}+\cdots-216 x+1$ & $3^{576} 7^{135}$ \\
\hline 5 & 162 & 3 & $x^{486}-486 x^{484}+\cdots+648 x+1$ & $3^{2187} 7^{405}$ \\
\hline$n$ & $k$ & $\mu$ & $D_{n}^{4^{2}, 3}(x)$, inherited from $P_{4,2}\left[T_{3}(x)\right]$ & $\Delta$ \\
\hline 1 & 8 & 2 & $x^{16}+x^{15}+\cdots-16\left(x^{2}+x\right)+1$ & $3^{8} 17^{15}$ \\
\hline 2 & 24 & 2 & $x^{48}-48 x^{46}+x^{45}+\cdots+48 x+1$ & $3^{72} 17^{45}$ \\
\hline 3 & 72 & 2 & $x^{144}-144 x^{142}+\cdots-144 x+1$ & $3^{360} 17^{135}$ \\
\hline 4 & 216 & 2 & $x^{432}-432 x^{430}+\cdots+432 x+1$ & $3^{1512} 17^{405}$ \\
\hline$n$ & $k$ & $\mu$ & $D_{n}^{5^{1}, 3}(x)$, inherited from $P_{5,1}\left[T_{3}(x)\right]$ & $\Delta$ \\
\hline 1 & 5 & 2 & $x^{10}+x^{9}+\cdots+12\left(x^{2}+x\right)+1$ & $3^{5} 11^{9}$ \\
\hline 2 & 15 & 2 & $x^{30}-30 x^{28}+\cdots-36 x+1$ & $3^{45} 11^{27}$ \\
\hline 3 & 45 & 2 & $x^{90}-90 x^{88}+\cdots+108 x+1$ & $3^{225} 11^{81}$ \\
\hline 4 & 135 & 2 & $x^{270}-270 x^{268}+\cdots-324 x+1$ & $3^{945} 11^{243}$ \\
\hline
\end{tabular}

size of the discriminants is huge and their dependence on just a few small primes is the happy circumstance that allows their factorization to be accomplished. As discussed by Brent [20], the factorization of integers with sizes comparable to the largest discriminants in the tables here poses computational difficulties which are still beyond technological capabilities of modern algorithms and hardware.

Table III shows some elements of hierarchies obtained from $P_{1}(x)$ with $T_{5}(x), T_{7}(x)$, and $T_{9}(x)$, where $T_{\alpha}(x)$ $=T_{\alpha}(x, A) \equiv(x+R / 2)^{\alpha}+(x-R / 2)^{\alpha}$, and $R=\sqrt{x^{2}-4 A}$ or, equivalently, $\quad T_{1}(x, A)=x, \quad T_{2}(x, A)=x^{2}-2 A, \quad$ and $T_{\alpha+1}(x, A)=x T_{\alpha}(x, A)-A T_{\alpha-1}(x, A)$, for $\alpha \geqslant 2$. The discriminants are vital for the analytical construction of orbital points but we cannot go into this here.

How about arbitrary values of $a$ ? Then $\left\{T_{\alpha}(x)\right\}$ produces a more elaborate set of polynomials and a more symmetrical hierarchical organization which for $a=2$ reduces to an orbital structuring distinct from that discussed above, involving products of different factors. Illustrative examples, showing the first members of four generalized hierarchies $\mathcal{D}_{n}(x)$, obtained with $T_{3}(x)$ and $T_{5}(x)$ from $P_{1}(x ; a)=x^{2}+x-a$ and $P_{2}(x ; a)=x^{2}-x-a+1$ for arbitrary values of $a$ and for $a$ $=2$, are the following:

$$
\begin{gathered}
\mathcal{D}_{2}^{1,3}(x ; a)=x^{6}-6 x^{4}+x^{3}+9 x^{2}-3 x-a, \\
\mathcal{D}_{2}^{1,3}(x ; 2)=(x+2)(x-1)^{2}\left(x^{3}-3 x-1\right) ; \\
\mathcal{D}_{2}^{2,3}(x ; a)=x^{6}-6 x^{4}-x^{3}+9 x^{2}+3 x-a+1,
\end{gathered}
$$

TABLE III. Examples of infinite hierarchies with two distinct

\begin{tabular}{|c|c|c|c|c|}
\hline$n$ & $k$ & $\mu$ & $D_{n}^{1,5}(x)$, inherited from $P_{1}\left[T_{5}(x)\right]$ & $\Delta$ \\
\hline \multirow[t]{2}{*}{1} & 2 & 1 & $x^{2}-x-1$ & 5 \\
\hline & 4 & 1 & $x^{4}+x^{3}-4\left(x^{2}+x\right)+1$ & $3^{2} 5^{3}$ \\
\hline \multirow[t]{2}{*}{2} & 10 & 1 & $x^{10}-10 x^{8}+\cdots+25 x^{2}-5 x-1$ & $5^{17}$ \\
\hline & 20 & 1 & $x^{20}-20 x^{18}+\cdots-100 x^{2}-20 x+1$ & $3^{10} 5^{35}$ \\
\hline \multirow[t]{2}{*}{3} & 50 & 1 & $x^{50}-50 x^{48}+\cdots+625 x^{2}-25 x-1$ & $5^{137}$ \\
\hline & 100 & 1 & $x^{100}-100 x^{98}+\cdots-100 x+1$ & $3^{350} 5^{275}$ \\
\hline \multirow[t]{2}{*}{4} & 250 & 1 & $x^{250}-250 x^{248}+\cdots-125 x-1$ & $5^{937}$ \\
\hline & 500 & 1 & $x^{500}-500 x^{498}+\cdots-500 x+1$ & $3^{250} 5^{1875}$ \\
\hline$n$ & $k$ & $\mu$ & $D_{n}^{1,7}(x)$, inherited from $P_{1}\left[T_{7}(x)\right]$ & $\Delta$ \\
\hline \multirow[t]{2}{*}{1} & 3 & 1 & $x^{3}-x^{2}-2 x+1$ & $7^{2}$ \\
\hline & 6 & 1 & $x^{6}+x^{5}-6\left(x^{4}+x^{3}\right)+8\left(x^{2}+x\right)+1$ & $3^{3} 7^{5}$ \\
\hline \multirow[t]{2}{*}{2} & 21 & 1 & $x^{21}-21 x^{19}+\cdots-49 x^{2}+14 x+1$ & $7^{38}$ \\
\hline & 42 & 1 & $x^{42}-42 x^{40}+\cdots+392 x^{2}-56 x+1$ & $3^{21} 7^{77}$ \\
\hline \multirow[t]{2}{*}{3} & 147 & 1 & $x^{147}-147 x^{145}+\cdots-98 x+1$ & $7^{416}$ \\
\hline & 294 & 1 & $x^{294}-294 x^{292}+\cdots+392 x+1$ & $3^{147} 7^{833}$ \\
\hline$n$ & $k$ & $\mu$ & $D_{n}^{1,11}(x)$, inherited from $P_{1}\left[T_{11}(x)\right]$ & $\Delta$ \\
\hline \multirow[t]{3}{*}{1} & 5 & 1 & $x^{5}-x^{4}-4 x^{3}+3 x^{2}+3 x+1$ & $11^{4}$ \\
\hline & 5 & 2 & $x^{10}+x^{9}-10\left(x^{8}+x^{7}\right)+34\left(x^{6}+x^{5}\right)$ & \\
\hline & & & $-43\left(x^{4}+x^{3}\right)+12\left(x^{2}+x\right)+1$ & $3^{5} 11^{9}$ \\
\hline \multirow[t]{2}{*}{2} & 55 & 1 & $x^{55}-55 x^{53}+\cdots+363 x^{2}-33 x-1$ & $11^{104}$ \\
\hline & 55 & 2 & $x^{110}-110 x^{108}+\cdots-132 x+1$ & $3^{55} 11^{209}$ \\
\hline
\end{tabular}
branches at every new generation. The distinct branches involve discriminants of two flavors only.

$$
\begin{aligned}
\mathcal{D}_{2}^{2,3}(x ; 2)= & \left(x^{2}-x-1\right)\left(x^{4}+x^{3}-4 x^{2}-4 x+1\right) \\
\mathcal{D}_{2}^{1,5}(x ; a)= & x^{10}-10 x^{8}+35 x^{6}+x^{5}-50 x^{4}-5 x^{3} \\
& +25 x^{2}+5 x-a \\
\mathcal{D}_{2}^{1,5}(x ; 2)= & (x+2)(x-1)\left(x^{2}-x-1\right)^{2} \\
& \times\left(x^{4}+x^{3}-4 x^{2}-4 x-1\right) \\
\mathcal{D}_{2}^{2,5}(x ; a)= & x^{10}-10 x^{8}+35 x^{6}-x^{5}-50 x^{4}+5 x^{3} \\
& +25 x^{2}-5 x-a+1 \\
\mathcal{D}_{2}^{2,5}(x ; 2)= & x^{10}-10 x^{8}+35 x^{6}-x^{5}-50 x^{4}+5 x^{3} \\
+ & 25 x^{2}-5 x-1 .
\end{aligned}
$$

Notice that $\mathcal{D}_{2}^{1,3}(x ; a)=\left[T_{3}(x)-z_{+}\right]\left[T_{3}(x)-z_{-}\right]$, where $z_{ \pm}=-1 \pm \sqrt{4 a+1}$ and, in general, that

$$
\mathcal{D}_{n}^{k^{j}, \alpha}(x ; a)=\prod_{i}\left[T_{\alpha}(x)-z_{i}\right]
$$

where the product runs over all zeros $z_{i}$ of $P_{k, j}(x)$. These remarkably simple decompositions allow orbital points for every $\mathcal{D}_{n}^{k^{j}, \alpha}(x)$ to be found analytically rather easily, the symmetrical decomposition persisting when the periodicity $k$ 
and/or $\alpha$ increase. By suitably sequencing functional compositions one sees that parameter-dependent daughters $\mathcal{D}_{n}^{k^{j}, \alpha}(x ; a)$ generate non-unique hierarchical substructurings.

How general are the orbital transformations discussed so far? We now show that they are generic properties of Abelian equations [15] and, consequently, of any orbital equation generated by iteration of rational functions since such equations are Abelian by construction.

Consider an irreducible orbital equation $P(x)$ having as its $k$ roots the set $\left\{x_{i}\right\}$ formed with the orbital points of a $k$-periodic orbit generated by iterating some rational dynamical system [16] $f(x)$ and labeled as usual

$$
x_{t+1}=f\left(x_{t}\right)
$$

with

$$
x_{k+1}=f\left(x_{k}\right) \equiv x_{1},
$$

where $t=1,2, \ldots, k$. Now, we use some rational function $\Theta(x)$ [thought as generalizing the previous $T_{\alpha}(x)$ ] to transform the orbital points $\left\{x_{i}\right\}$ into a new set $\left\{\xi_{i}\right\}$ as follows:

$$
\xi_{1}=\Theta\left(x_{1}\right), \quad \xi_{2}=\Theta\left(x_{2}\right), \quad \cdots, \quad \xi_{k}=\Theta\left(x_{k}\right),
$$

and with these $\xi_{i}$ build a new orbital equation $Q(\xi)$ having them as roots. Then, straightforward computations show that it is always possible to select suitable branches (Riemann sheets) which connect back conformally the orbital points in $\left\{\xi_{i}\right\}$ with the original points in $\left\{x_{i}\right\}$, preserving the ordering, through a computable function, say $g(\xi)$, such that

$$
x_{t}=g\left(\xi_{t}\right)=g\left[\Theta\left(x_{t}\right)\right],
$$

and, consequently,

$$
\xi_{t+1}=\Theta\left(x_{t+1}\right)=\Theta\left[f\left(x_{t}\right)\right]=\Theta\left\{f\left[g\left(\xi_{t}\right)\right]\right\} \equiv \varphi\left(\xi_{t}\right) .
$$

This passage from the dynamical system $x_{t+1}=f\left(x_{t}\right)$ to the new one $\xi_{t+1}=\varphi\left(\xi_{t}\right)$ establishes the following useful theorem: A rational transformation of an Abelian equation is an Abelian equation or, more physically, rational transformations of orbital equations are orbital equations. The particular periods which are thereby interconnected will depend on the reducibility of $g(\xi)$, more precisely, on its splitting field [18]. This quite general function $g(\xi)$, the "transformation motor," is responsible for all hierarchical structuring of orbits discussed here and more [21].

In conclusion, infinite hierarchies of inherited orbits is a generic property of orbital equations generated by iteration of rational functions. The key to inheritance is the composition of functions indicated in Eq. (14), involving transformations, i.e., automorphisms, of the orbital points. The set $\{\Theta(x)\}$ and the seeds underlying every hierarchy provide the minimal fiducial information, the genetic keys, necessary to unfold the dynamics into interesting orbital structures ready to be explored. A promising possibility is to investigate the impact of the orbit-within-orbit structuring in cycle expansions of Ruelle's dynamical zeta function and in trace formulas popular nowadays in atomic physics [6]. Another, the classification of "shrimps" [22], i.e., of the nucleation of stability islands in dissipative systems.

I thank Professor João Corte-Real, Universidade de Lisboa, for his kind interest in this work and for many helpful discussions, and Dr. Ulrich Wenschuh, Forschungszentrum Jülich, for kindly sending me a copy of Abel's paper. This work was partially supported by the program Praxis XXI (Portugal) and CNPq (Brazil).
[1] F. Diakonos, P. Schmelcher, and O. Biham, Phys. Rev. Lett. 81, 4349 (1998).

[2] T. L. Carroll, Phys. Rev. E 59, 1615 (1999).

[3] P. Schmelcher and F. Diakonos, Phys. Rev. E 57, 2739 (1998).

[4] M. C. Gutzwiller, Chaos in Classical and Quantum Mechanics (Springer, NY, 1990); R. Balian and C. Bloch, Ann. Phys. (N.Y.) 69, 76 (1972); 85, 514 (1974); M. V. Berry, Proc. R. Soc. London, Ser. A 143, 183 (1987).

[5] M. Combescure, J. Ralston, and D. Robert, Commun. Math. Phys. 202, 463 (1999).

[6] Several interesting review articles appear in Supersymmetry and Trace Formulae: Chaos and Disorder edited by V. I. Lerner, J. P. Keating, and D. E. Khmelnitskii, NATO ASI Series 370 (Kluwer, New York, 1999); C. P. Dettmann and G. P. Morriss, Phys. Rev. Lett. 78, 4201 (1997).

[7] A. N. Sharkosvkii, Ukr. Math. 16, 61 (1964).

[8] T.-Y. Li and J. A. Yorke, Am. Math. Monthly 82, 985 (1975).

[9] E. Bertram and P. Horák, Math. Intelligencer 21(3), 6 (1999).

[10] W. P. Thurston, Bull. Am. Math. Soc. 19, 417 (1988).

[11] J. M. Gambaudo, S. van Strier, and C. Tresser, C.R. Acad. Sci.
Paris I 310, 291 (1990).

[12] T. Hall, Nonlinearity 7, 367 (1994).

[13] J. A. C. Gallas, Europhys. Lett. 47, 649 (1999).

[14] We call a $k$-periodic orbit "genuine" if all its $k$ orbital points are different among themselves. In this paper, "periodic orbit" always means a genuine periodic orbit.

[15] See G. B. Mathews, in Algebraic Equations, 3rd ed., revised by W. E. H. Berwick (Cambridge University Press, Cambridge, 1930), Chap. 3, reprinted by Hafner, New York, 1960. Original reference: N. H. Abel, J. Reine Angew. Math. 4, 131 (1829).

[16] Our use of a one-dimensional dynamical system implies no restriction. Higher-dimensional systems can be always reduced to a one-dimensional equivalent by eliminating all but one variable among equations of motion.

[17] To simplify, we work with $P_{1}(x)$ instead of $P_{1,1}(x)$ and $P_{1,2}(x)$.

[18] I. Stewart, Galois Theory, 2nd ed. (Chapman and Hall, London, 1994).

[19] Inheritance from $P_{1}\left[T_{3}(x)\right]$ requires $D_{0}(x)=x-1$. This nonessential exception is a simple consequence of working with $P_{1}(x)$ instead of $P_{1,1}(x)$ and $P_{1,2}(x)$. 
[20] R. P. Brent, Math. Comput. 68, 429 (1999); V. Trevisan and J. B. Carvalho, J. Supercomputing 9, 179 (1995).

[21] J. A. C. Gallas, Physica A 283, 17 (2000).

[22] J. A. C. Gallas, Phys. Rev. Lett. 70, 2714 (1993); Appl. Phys.
B: Lasers Opt. 60, S-203 (1995); M. W. Beims and J. A. C. Gallas, Physica A 238, 225 (1997); B. R. Hunt, J. A. C. Gallas, C. Grebogi, J. A. Yorke, and H. Koçak, Physica D 129, 35 (1999). 